\title{
Modulation of Insulin Gene Expression with CRISPR/Cas9-based Transcription Factors
}

Bakhytzhan Alzhanuly ${ }^{1,2,3 *}$, Zhussipbek Y. Mukhatayev ${ }^{1,2} \mathbb{D}$, Dauren M. Botbayev ${ }^{1,2} \mathbb{D}, \quad$ Yeldar Ashirbekov ${ }^{1}$, Nurlybek D. Katkenov ${ }^{4}$ (iD, Nurlan T. Dzhaynakbaev ${ }^{5}$, Kamalidin O. Sharipov ${ }^{1}$ (D)

${ }^{1}$ Aitkhozhin Institute of Molecular Biology and Biochemistry, Almaty, Kazakhstan; ${ }^{2}$ Department of Molecular Biology and Genetics, Faculty of Biology and Biotechnology, Al-Farabi Kazakh National University, Almaty, Kazakhstan; ${ }^{3}$ Department of Research and Development, Almaty Management University, Almaty, Kazakhstan; ${ }^{4}$ Zhangir Khan University, Uralsk, Kazakhstan; ${ }^{5}$ KazakhRussian Medical University, Almaty, Kazakhstan

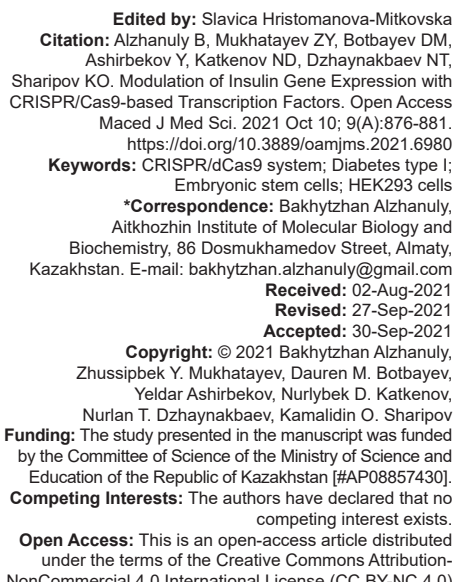

Edited by: Slavica Hristomanova-Mitkovska n: Alzhanuly B, Mukhatayev ZY, Botbayev DM Ashirbekov Y, Katkenov ND, Dzhaynakbaev N Sharipov KO. Modulation of Insulin Gene Expression with CRISPR/Cas9-based Transcription Factors. Open Acces (A) :876-881. Keywords: CRISPR/dCas9 system; Diabetes type I Correspondence: Bakhytzhan Alzhanuly, *Correspondence: Bakhytzhan Alzhanuly,
Aitkhozhin Institute of Molecular Biology and Biochemistry, 86 Dosmukhamedov Street, Almaty, Kazakhstan. E-mail: bakhytzhan.alzhanuly@gmail.com Received: 02-Aug-202 Revised: 27-Sep-202 Accepted: $30-$ Sep-202 Copyright: @ 2021 Bakhylzhan Alzhanuly, hussipbek Y. Mukhatayev, Dauren M. Botbaye Nurlan T. Dzhaynakbaev, Kamalidin O. Sharipov Funding: The study presented in the manuscript was funded by the Committee of Science of the Ministry of Science and Education of the Republic of Kazakhstan [\#AP08857430]. Competing Interests: The authors have declared that no competing interest exists. Open Access: This is an open-access article distributed under the terms of the Creative Commons Attribution-

\begin{abstract}
BACKGROUND: The discovery and use of CRISPR/Cas9 technology have enabled researchers throughout the globe to continuously edit genomes for the benefit of science and medicine. Diabetes type I is one field of medicine where CRISPR/Cas9 has a strong potential for cell therapy development. The long-lasting paucity of healthy cells for clinical transplantation into diabetic patients has led to the search of new methods for producing $\beta$-cells from other human cell types. Embryonic stem cells are being studied worldwide as one most promising solution of this need.

AIM: The aim of the study is to check the feasibility of modulating human insulin transcription using CRISPR/Cas9based synthetic transcription regulation factors.

METHODS: A new approach for creating potential therapeutic donor cells with enhanced and suppressed insulin production based on one of the latest achievements of human genome editing was developed.

RESULTS: Both synthetic transcription activator (VP64) and transcription repressor (KRAB) proteins were shown to function adequately well as a part of the whole CRISPR/Cas9-based system.

CONCLUSION: We claim that our results have a lot to offer and can bring light to many studies where numerous
\end{abstract} laboratories are struggling on to treat this disease.

\section{Introduction}

Latest advances of developing synthetic gene regulation systems have led to massive discoveries in biomedical science. Research groups around the world have developed synthetic transcription factors that can regulate expression of various endogenous genes by targeting their promoters and enhancers. Until now, synthetic transcription factors have been used for fixing genetic mutations [1], activation of tumor suppressors [2], controlling stem cell differentiation [3], and stimulation of tissue regeneration [4].

To date, three genome editing technologies are available: (1) Transcription activator like effector nucleases (TALENs) [5], (2) zinc finger nucleases (ZFNs) [6], and (3) clustered regularly interspaced short palindromic repeats (CRISPR)/CRISPRassociated protein 9 (Cas9) [7]. These technologies, more CRISPR/Cas9, are carried out in studies where synthetic transcription factors are also involved [8], [9],
[10]. The advent of the genome editing technologies has addressed numerous issues in many therapeutic areas, but there has not been made much effort in using these techniques for treating diabetes. Cell therapy that is based on transplantation of donor cells is considered by many researchers in the field as a promising approach to treat diabetic patients, especially those with type I diabetes. Replacing lost $\beta$-cells with functional and healthy cells could be the future of the treatment. However, the lack of donor materials worldwide for clinical cell transplantation greatly limits this therapy [11], [12], thus, production of $\beta$-cells (or $\beta$-like cells) from other cell types is now considered as the most promising approach. One potential solution within the approach is the use of embryonic stem cells (ESCs). ESCs could be of unlimited supply of $\beta$-cells, but there is still no proper differentiation protocol although a number of research groups have been working on it for decades [13], [14] [15], [16]. But here as alternative, we offer an approach that has a potential to deliver $\beta$-cells from human cells different than ESCs. 
Insulin plays a key role in maintaining metabolic homeostasis of human body and regulation of its gene expression is in good accordance with the principle role of the hormone [17], [18]. The $\beta$-cells of the pancreas act as the exclusively main place where the mammalian insulin gene (INS) is expressed. The expression is very accurately handled by specific combinations and synergistic cooperativity of numerous transcription factors [18], [19]. Although modulation of various human genes has been performed in laboratories with the use of latest achievements in modern genome editing technologies [20], [21], none of such studies were devoted to in vitro controlling the expression of insulin gene, despite the critical significance of the hormone.

CRISPR/Cas9 technology consists of a gRNA molecule for targeting and Cas9 nuclease for cleavage. Cas9 comes from Streptococcus pyogenes and functions to protect the bacteria from foreign bodies by destroying their DNA [7]. In nature, these bacteria have two separate RNA molecules (crRNA - transactivating CRISPR RNA and pre-crRNA - precursor CRISPR RNA) that by acting together direct Cas9 to a target (for example, phage). However, in research, scientists usually synthetically join the two as one chimeric guide RNA (gRNA). The gRNA forms a ribonucleoprotein complex with the Cas9. A number of research laboratories have previously shown that expression of type // CRISPR/Cas9 system in mammalian cells leads to the formation of double-strand brakes (DSBs) at target sites: The gRNA's protospacer region matches with a $20 \mathrm{bp}$ sequence of the target DNA and as a result, Cas9 cleaves the DNA [7], [8].

In the past years, CRISPR/Cas9 system has gained superior attention: The target specificity of Cas9 derives from RNA: DNA complementarity while TALENs and ZFNs require modifications to the protein itself to target DNA [22]. CRISPR/Cas9 can be easily adapted to target any genomic sequence just by changing the protospacer of gRNA. The Cas9 protein component remains unchanged. This easy programmability of CRISPR/Cas 9 system is a significant advantage over ZFNs and TALENs, which require tremendous amount of bench work.

The aim of the study was to check the feasibility of modulating human insulin transcription using CRISPR/Cas9-based synthetic transcription regulation factors.

\section{Materials and Methods}

\section{Cell culture and transfections}

HEK 293 cells were obtained from the Core Facility of Asfendiyarov Kazakh National Medical University (Almaty) and seeded in 6-well plate to be $80 \%$ confluent at the time of transfection. The cells were maintained in Dulbecco's Modified Eagle's Medium (DMEM, Life Technologies) that was supplemented with $10 \%$ heat-inactivated fetal bovine serum (FBS, Life Technologies) and 1\% penicillin/streptomycin (Life Technologies). $2.5 \mathrm{ug}$ of DNA, 5 ul of P3000 reagent, and 7.5 ul $(2 \times 3.75 \mathrm{ul})$ of lipofectamine 3000 reagent were used for each well, according to manufacturer's instructions (Invitrogen). Cells were cultured at $37^{\circ} \mathrm{C}$ with $5 \% \mathrm{CO}_{2}$. Forty-eight-72 h post-transfection cells were collected and RNA was harvested for analysis.

\section{Plasmids}

The plasmid encoding deactivated Cas9 (with H840A and D10A mutations) and VP64 that have been designed by Perez-Pinera et al. [23] were obtained from Addgene (\#47107).

Following the algorithm that Doench et al. have developed [24], a number of gRNAs were designed according to the sequence surrounding the transcriptional start site of INS gene. The plasmid pX330A_D10-1x4, which expresses native Cas9 nuclease and that was used for testing newly designed gRNAs, was also obtained from Addgene (\#58774). The gRNAs were cloned into the plasmid and the construct was then transfected into normal HEK293 cells and screened to identify the most effective gRNAs using "Surveyor" nuclease assay [25]. Eventually, the most effective two gRNAs were identified and cloned into pLV GG hUbC-dsRED plasmid, which was designed by Kabadi et al. [26] and available from Addgene (\#84034).

The plasmid expressing dCas9 $(\mathrm{D} 10 \mathrm{~A}+\mathrm{D} 839 \mathrm{~A}+\mathrm{H} 840 \mathrm{~A}+\mathrm{N} 863 \mathrm{~A})$ linked to KRAB repressor domain [27] was also obtained from Addgene (\#110820).

\section{Lentivirus production}

Briefly, 2.5 million HEK293T cells were plated per $10 \mathrm{~cm}$ dish. The next day, cells were transfected by the calcium phosphate transfection method with $5 \mathrm{ug}$ of transfer (expression) vector, 6 ug of packaging plasmid, and $33 \mathrm{ul}$ of lipofectamine 3000 transfection reagent, according to manufacturer's instructions (Invitrogen). All the components were pre-diluted in serum-free Opti-MEM media before the final mix was added onto the cells. Then, the cells were incubated at $37^{\circ} \mathrm{C}$ with $5 \% \quad \mathrm{CO}_{2}$ and the media were changed after $12-18 \mathrm{~h}$. The viral supernatant was collected 24 and $48 \mathrm{~h}$ after each media change, passed through a 0.45 um filter, tittered, aliquoted ( $35 \mathrm{ul}$ ), and stored at $-80^{\circ} \mathrm{C}$ for the transduction procedure.

\section{Transduction}

HEK293 cells (with dCas9 and VP64) were plated in 12-well plate. Polybrene stock $(10 \mathrm{mg} / \mathrm{ml})$ was diluted as $1 / 1000$ in $1 \mathrm{ml}$ of DMEM media (with $10 \%$ 
FBS and $1 \%$ penicillin/streptomycin). A total volume of pre-made lentivirus (35 ul) were added to the mixture. The total mixture (DMEM + polybrene + lentivirus) was added to each well. Cells were incubated at $37^{\circ} \mathrm{C}$ with $5 \% \mathrm{CO}_{2}$, analyzed under fluorescent microscope (2-3 days) and flow cytometry (2 days). Next, RNA was harvested for qPCR.

\section{Flow cytometry}

Forty-eight hours post-transduction cells were assayed. The cells were detached from the wells using $0.5 \%$ trypsin (Invitrogen) and pre-stained with LIVE/DEAD Fixable Near IR Dead Cell dye, according to manufacturer's instructions (Thermo Fisher Scientific). Transduced cells were gated on dsRED expressing cells. Stained cells were analyzed using a BD FACSymphony flow cytometer and FlowJo v10.3.0 software (FlowJo LLC, OR, USA).

\section{Quantitative real-time polymerase chain reaction (qPCR)}

Total RNA was isolated using the miRNeasy Mini RNA isolation kit (Qiagen) according to manufacturer's instructions where DNAse digestion was done using the DNA-free Kit (Applied Biosystems). One ug of RNA was used for complementary DNA (cDNA) synthesis which was performed using SuperScript VILO cDNA Synthesis Kit (Invitrogen). Synthesized cDNA samples were diluted $1 / 10$ times in $\mathrm{ddH}_{2} \mathrm{O}$. All the qPCRs were performed using PerfeCTa SYBR Green FastMix (Quanta Biosciences) in CFX96 Real-Time PCR Detection System (Bio-Rad). The results were expressed as a relative fold increase in mRNA expression of the gene of interest normalized to housekeeping gene (Actin) expression using the $2^{-\Delta \Delta C t}$ method.

\section{Statistical analysis of data}

Statistical analysis was performed using GraphPad Prism 8.0 software (GraphPad Software Inc., CA, USA). Data are presented as bars and dot plots with mean values \pm standard deviation. The data were evaluated by one-way ANOVA analysis of variance accounting for different variances across the groups, with post hoc Tukey's or Sidak's multiple comparisons test. Statistical significance is represented as ${ }^{*} p<0.05$, ${ }^{* *} p<0.01,{ }^{* * *} p<0.001$, or ${ }^{* * * *} p<0.0001$.

\section{Results}

To achieve the goal, we first obtained a line of HEK 293 cells that would stably express the deactivated form of the Cas9 nuclease (dCas9) and well-known transcription activator - VP64 protein. As there was no need to cleave the target DNA in the experiments, dCas 9 was used instead of natural Cas9. VP64, linked to the nuclease, acts as an activator of transcription when brought close to the promoter of an endogenous gene. The dCas 9 has been designed earlier by Jinek et al. [7] through inducing point mutations into the catalytic amino acid residues (D10A, H840A) of Cas9. In general, natural HEK 293 cells were transfected with the dCas9-VP64 plasmid and the expressions of the proteins were studied. Sufficient expressions were achieved in the transfected cells (significant $p=0.0257$ and 0.0008 for dCas9 and VP64, respectively; Figure $\mathrm{S} 1$ in the Supplementary Materials).

We next obtained our vector construct for cloning and expressing gRNAs by modifying the previously obtained lentiviral plasmid. Earlier, synergistic effect of several gRNAs (in some cases up to 4) targeted to the same transcriptional site has been shown to significantly enhance the overall efficiency of endogenous gene activation compared to when a single gRNA is used [20]. Therefore, following the algorithm that has previously been developed [24], several INS gRNAs were designed, tested, and the best ones were cloned into the lentiviral plasmid.

Next, the obtained gRNA carrying plasmid was packaged into lentivirus using lentiviral production method [28]. Concentrated viral material was then used to infect targeting INS gene in the previously produced dCas9-VP64 HEK 293 cells. Lentiviral gene construct encoded with insulin was successfully transduced with $43.7 \%$ efficiency into host cells (Figure 1 ).

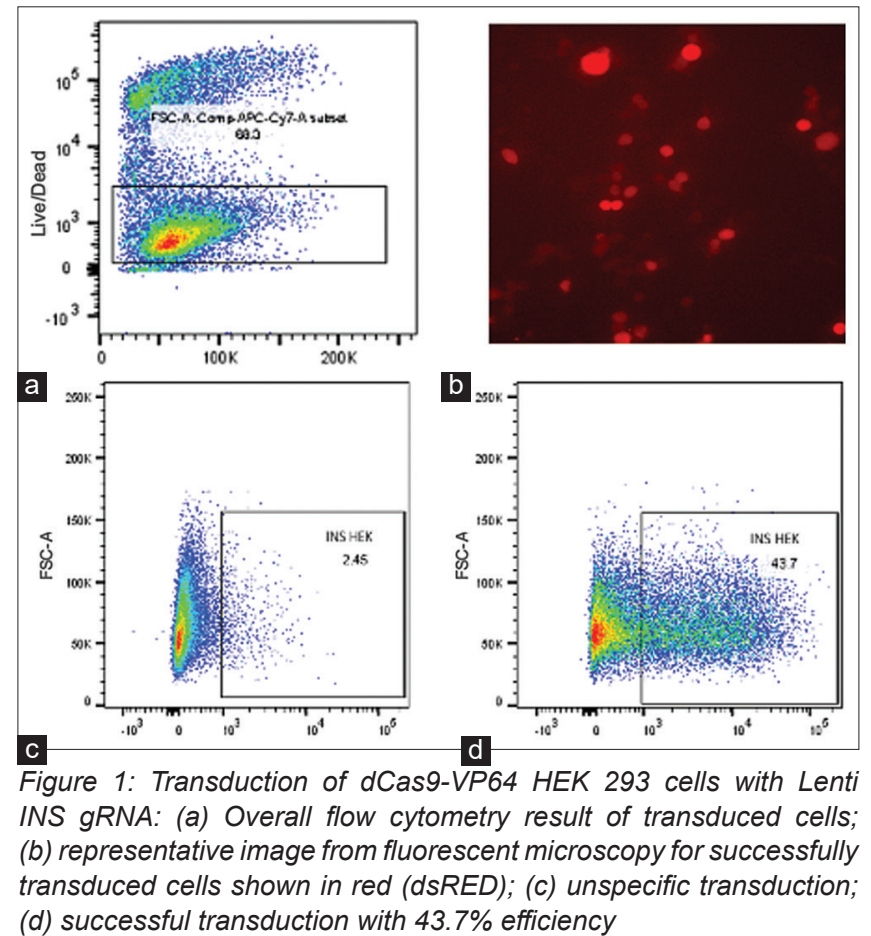

As next tested by qPCR, the inserted gRNAs were properly expressed as shown by significant 
fold increase (over 1000x) in INS transcription in test samples compared with negative controls (Figure 2). However, this increase was fairly less than in naturally insulin expressing Min6 cells (nearly 5x).

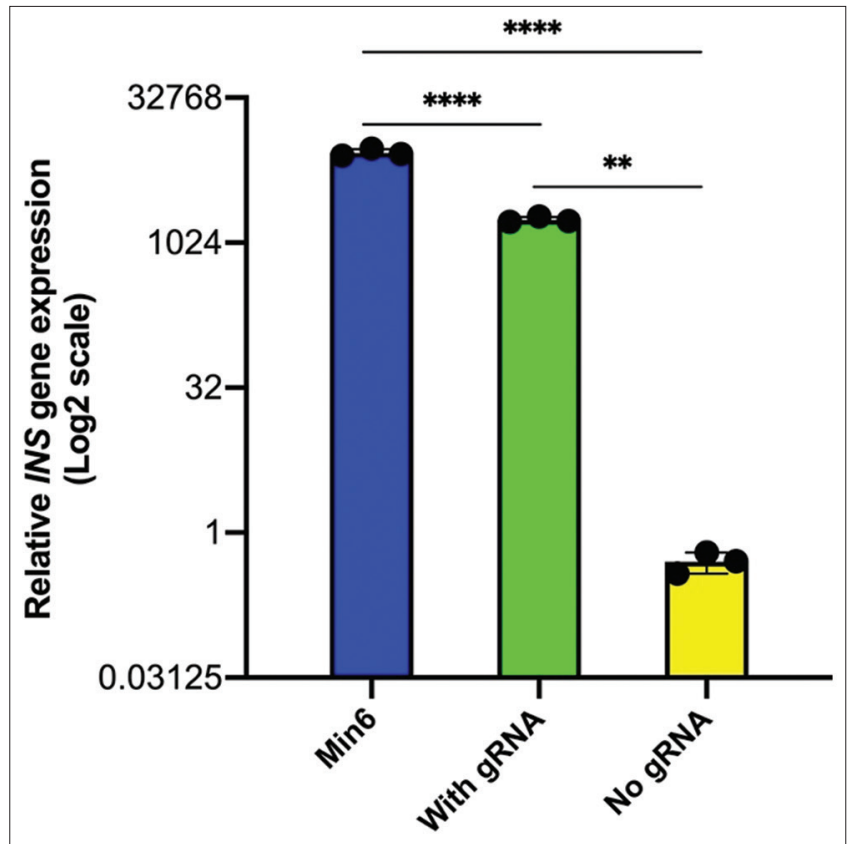

Figure 2: INS gene activation with gRNA-directed VP64 transcription factor fused to dCas9: Min6 - naturally insulin-producing mouse cell line (positive control); with gRNA - dCas9-VP64 HEK 293 cells with activated INS gene; no gRNA - only dCas9-VP64 HEK 293 cells. Statistical analysis was performed by a one-way ANOVA test followed by Tukey's post hoc test for multiple comparisons. ${ }^{* \star} p<0.01$; ${ }^{* \star * \star} p<0.0001$

After successful activation of the target gene, the next aim was to check if it would be feasible to further manipulate it. In particular, it was sought to lower the recently activated INS transcription as a possible benefit for diabetes research. Several studies have previously showed earlier that Kruppel-associated box (KRAB) protein could be as a strong transcription inhibitor for most endogenous genes [27], [29]. Following this, the HEK 293 cells with activated insulin gene (dCas9VP64+INS gRNA) were transfected with the previously obtained plasmid with dCas9-KRAB complex cloned in. The latter dCas 9 had a slightly different amino acid sequence than the previous one, but can bind with the INS gRNAs which are still expressed by the host cell. As a result of the transfection, substantially lower amount of insulin mRNA (nearly 1000x) was observed in the infected cells than in control samples (no dCas9KRAB addition, Figure 3). In addition, as expected, transfected cells had well expressed dCas 9 and KRAB (Figure S3 in the Supplementary Materials).

\section{Discussion}

The study describes gRNA-assisted endogenous insulin transcription activation and its repression in normal HEK 293 cells. Purposefully designed gRNAs, together with deactivated Cas9 nuclease (dCas9) linked to synthetic transcription factors (VP64 and KRAB domains), adequately regulated the expression of INS gene. In addition, synergistic effect of several gRNAs (in this case 2), targeted to the same sequence, was properly validated.

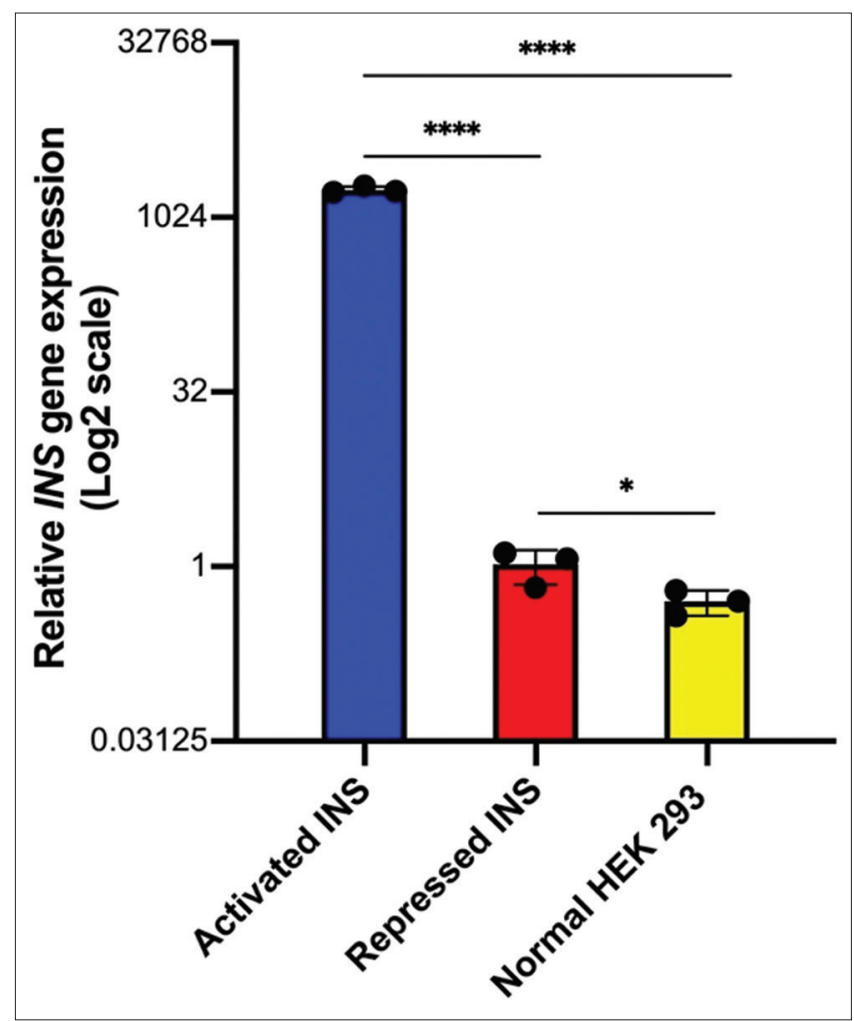

Figure 3: Repression of activated INS gene in dCas9-VP64 HEK 293 cells: gRNA + (dCas9-VP64) - positive control; gRNA + (dCas9-VP64) $+(d$ Cas9-KRAB) - cells after transfection with the repression domain plasmid; natural HEK 293 cells - negative control. Statistical analysis was performed by a one-way ANOVA test followed by Tukey's post hoc test for multiple comparisons. ${ }^{*} p<0.05 ;{ }^{* \star * *} p<0.0001$

The purpose for repressing insulin production came out of two reasons: (1) Studies have reported that the burden of producing relatively extensive supply of insulin significantly delays the natural rate of $\beta$-cell proliferation [30] and negatively affects the differentiation of $\beta$-cells [31] even under normal physiological conditions; (2) there are a number of synthetic transcription repressor proteins available, and our experiments were as a specific case for validating their function for regulation of insulin transcription. As the results show, the repression is feasible and our findings shed light on potential future experiments where controlled differentiation of embryonic stem cells into $\beta$-cells with desired level of insulin synthesis can potentially be performed. In addition, it is assumed that modulating the level of insulin production during the differentiation stages might be advantageous in future experiments.

In the inhibition experiment, the result that the gRNAs bound better with the second dCas9 than the first initial sample (in the dCas9-VP64 complex) can be 
studied more. One suggestion might be in the amino acid sequences of the nucleases that a few changes could lead to that significant difference in binding. Although the experiment was performed more than once, very similar results were received each time.

More experiments might be needed to study possible off-target effects of CRISPR/Cas9-based regulation of insulin transcription. As was mentioned earlier, regulation of INS gene is sufficiently complicated and therefore this modulation might have some off-target consequences. A study found that RNA-guided dCas9KRAB transcription repression introduced minimal changes into the gene expression patterns of human cells [32]. It's also critical whether or not the dCas9 fusions with synthetic proteins affect the specificity of the CRISPR/Cas9 system. In addition, most of RNA-assisted endonucleases, including Cas9, are very effective and easy programmable DNA-altering molecules, but because of their hereditary organization and mechanism, these nucleases have more serious off-target effects than ZFNs and TALENs [33]. Therefore, TALENs and ZFNs could be studied as well to find out which of three genome editing technologies give the best result exclusively for the modification of insulin gene expression.

Finally, long time survival and possible glucose-responsive functions of the manipulated cells can be further observed. Effective control of the insulin gene behavior after enhancing as well as lowering its transcription is also of critical importance.

\section{Conclusions}

Our findings can serve as proof of principle that CRISPR/dCas9 system works acceptably well even when several heterologous synthetic regulator proteins are linked to the dCas9 nuclease. These results now open avenue for the possibility of other transcription regulator molecules (e.g. DNA methylation modifiers, novel effector enzymes) to be fused to dCas 9 for new research perspectives in the area. Ultimately, these results should enable and implement other future applications of CRISPR/dCas 9 system for the development of cell therapies against diabetes type I and for medicine in general.

\section{Funding Statement}

The study presented was funded by the Committee of Science of the Ministry of Science and Education of the Republic of Kazakhstan [Grant number \#AP08857430].

\section{Data Availability}

All supporting data are available from the corresponding author on request.

\section{References}

1. Hussey BJ, McMillen DR. Programmable T7-based synthetic transcription factors. Nucleic Acids Res. 2018;46(18):9842-54. http://doi.org/10.1093/nar/gky785

PMid:30169636

2. Beltran AS, Russo A, Lara H, Fan C, Lizardi PM, Blancafort $P$. Suppression of breast tumor growth and metastasis by an engineered transcription factor. PLoS One. 2011;6(9):e24595. http://doi.org/10.1371/journal.pone.0024595 PMid:21931769

3. Wada T, Wallerich S, Becskei A. Synthetic transcription factors switch from local to long-range control during cell differentiation. ACS Synth Biol. 2019;8(2):223-31. https://doi.org/10.1021/ acssynbio.8b00369

4. Rebar EJ, Huang Y, Hickey R, Nath AK, Meoli D, Nath S, et al Induction of angiogenesis in a mouse model using engineered transcription factors. Nat Med. 2002;8(12):1427-32. https://doi. org/10.1038/nm1202-795 PMid:12415262

5. Zhang F, Cong L, Lodato S, Kosuri S, Church GM, Arlotta P. Efficient construction of sequence-specific TAL effectors for modulating mammalian transcription. Nat Biotechnol. 2011;29(2):149-53

6. Beerli RR, Barbas $\mathrm{CF} 3^{\text {rd }}$. Engineering polydactyl zinc-finger transcription factors. Nat Biotechnol. 2002;20(2):135-41. https:// doi.org/10.1038/nbt0202-135

PMid: 11821858

7. Jinek $M$, Chylinski $K$, Fonfara I, Hauer $M$, Doudna JA, Charpentier E. A programmable dual-RNA-guided DNA endonuclease in adaptive bacterial immunity. Science. 2012;337(6096):816-21. https://doi.org/10.1126/ science. 1225829

PMid:22745249

8. Mali P, Yang L, Esvelt KM, Aach J, Guell M, DiCarlo JE, et al. RNA-guided human genome engineering via Cas9. Science. 2013;339(6121):823-6. https://doi.org/10.1126/ science. 1232033

PMid:23287722

9. Matjusaitis M, Wagstaff LJ, Martella A, Baranowski B, Blin C, GogolokS, etal. Reprogramming of fibroblasts to oligodendrocyte progenitor-like cells using CRISPR/Cas9-based synthetic transcription factors. Stem Cell Rep. 2019;13(6):1053-67. https://doi.org/10.1016/j.stemcr.2019.10.010 PMid:31708478

10. Mol M, Kabra R, Singh S. Genome modularity and synthetic biology: Engineering systems. Prog Biophys Mol Biol. 2018;132:43-51. https://doi.org/10.1016/j. pbiomolbio.2017.08.002 PMid:28801037

11. Pepper AR, Gala-Lopez B, Ziff O, Shapiro AJ. Current status of clinical islet transplantation. World J Transplant. 2013;3(4):48-53. https://doi.org/10.5500/wjt.v3.i4.48

PMid:24392308 
12. Dinnyes A, Schnur A, Muenthaisong S, Bartenstein P, Burcez CT, Burton $\mathrm{N}$, et al. Integration of nano-and biotechnology for betacell and islet transplantation in Type-1 diabetes treatment. Cell Prolif. 2020;53(5):e12785. https://doi.org/10.1111/cpr.12785

13. Kroon E, Martinson LA, Kadoya K, Bang AG, Kelly OG, Eliazer $\mathrm{S}$, et al. Pancreatic endoderm derived from human embryonic stem cells generates glucose-responsive insulinsecreting cells in vivo. Nat Biotechnol. 2008;26(4):443-52. https://doi.org/10.1038/nbt1393

PMid: 18288110

14. Pagliuca FW, Millman JR, Gurtler M, Segel M, Van Dervort A, $\mathrm{Ryu} \mathrm{JH}$, et al. Generation of functional human pancreatic beta cells in vitro. Cell 2014;159(2):428-39. https://doi.org/10.1016/j. cell.2014.09.040

PMid:25303535

15. Nostro MC, Sarangi F, Yang C, Holland A, Elefanty AG, Stanley EG, et al. Efficient generation of NKX6-1+ pancreatic progenitors from multiple human pluripotent stem cell lines. Stem Cell Reports. 2015;4(4):591-604. https://doi.org/10.1016/j. stemcr.2015.02.017 PMid:25843049

16. Millman JR, Xie C, Van Dervort A, Gürtler M, Pagliuca FW, Melton DA. Generation of stem cell-derived beta-cells from patients with Type 1 diabetes. Nat Commun. 2016;7:11463. https://doi.org/10.1038/ncomms 11463

\section{PMid:27163171}

17. Poitout V, Hagman D, Stein R, Artner I, Robertson RP, Harmon JS Regulation of the insulin gene by glucose and fatty acids. J Nutr. 2006;136(4):873-6. https://doi.org/10.1093/jn/136.4.873 PMid: 16549443

18. Kuroda A, Rauch TA, Todorov I, Ku HT, Al-Abdullah IH, Kandeel F, et al. Insulin gene expression is regulated by DNA methylation. PLoS One. 2009;4(9):e6953. https://doi.org/10.1371/journal. pone.0006953

PMid:19742322

19. Melloul D, Marshak S, Cerasi E. Regulation of insulin gene transcription. Diabetologia. 2002;45(3):309-26. https://doi. org/10.1007/s00125-001-0728-y PMid:11914736

20. Maeder ML, Linder SJ, Reyon D, Angstman JF, Fu Y, Sander JD, et al. Robust, synergistic regulation of human gene expression using TALE activators. Nat Methods. 2013;10(3):243-5. https:// doi.org/10.1038/nmeth.2366 PMid:23396285

21. Yin $\mathrm{H}$, Song $\mathrm{CQ}$, Suresh $\mathrm{S}$, Kwan $\mathrm{SY}, \mathrm{Wu} \mathrm{Q}$, Walsh $\mathrm{S}$, et al. Partial DNA-guided Cas 9 enables genome editing with reduced off-target activity. Nat Chem Biol. 2018;14(3):311-6. https://doi. org/10.1038/nchembio.2559

PMid:29377001

22. Mali P, Esvelt KM, Church GM. Cas9 as a versatile tool for engineering biology. Nat Methods. 2013;10(10):957-63. https:// doi.org/10.1038/nmeth.2649

PMid:24076990

23. Perez-Pinera P, Kocak DD, Vockley CM, Adler AF, Kabadi AM,
Polstein LR, et al. RNA-guided gene activation by CRISPR-Cas9based transcription factors. Nat Methods. 2013;10(10):973-6. https://doi.org/10.1038/nmeth.2600

PMid:23892895

24. Doench JG, Fusi N, Sullender M, Hegde M, Vaimberg EW, Donovan KF, et al. Optimized sgRNA design to maximize activity and minimize off-target effects of CRISPR-Cas9. Nat Biotechnol. 2016;34(2):184-91. https://doi.org/10.1038/nbt.3437 PMid:26780180

25. Guschin DY, Waite AJ, Katibah GE, Miller JC, Holmes MC, Rebar EJ. A rapid and general assay for monitoring endogenous gene modification. Methods Mol Biol. 2010;649:247-56. https:// doi.org/10.1007/978-1-60761-753-2_15 PMid:20680839

26. Kabadi AM, Ousterout DG, Hilton IB, Gersbach CA. Multiplex CRISPR/Cas9-based genome engineering from a single lentiviral vector. Nucleic Acids Res. 2014;42(19):e147. https:// doi.org/10.1093/nar/gku749

PMid:25122746

27. Yeo NC, ChavezA, Lance-Byrne A, Chan Y, Menn D, Milanova D, et al. An enhanced CRISPR repressor for targeted mammalian gene regulation. Nat Methods. 2018;15(8):611-6. https://doi. org/10.1038/s41592-018-0048-5

PMid:30013045

28. Wang $X$, McManus $M$. Lentivirus production. $J$ Vis Exp. 2009;32:1499. https://doi.org/10.3791/1499 PMid: 19801965

29. Rosenbluh J, Xu H, Harrington W, Gill S, Wang X, Vazquez F, et al. Complementary information derived from CRISPR Cas9 mediated gene deletion and suppression. Nat Commun. 2017;8:15403. https://doi.org/10.1038/ncomms15403 PMid:28534478

30. Song B, Scheuner D, Ron D, Pennathur S, Kaufman RJ. Chop deletion reduces oxidative stress, improves beta cell function, and promotes cell survival in multiple mouse models of diabetes. J Clin Invest. 2008;118(10):3378-89. https://doi.org/10.1172/ $\mathrm{JCl} 34587$

PMid: 18776938

31. Kaufman RJ, Back SH, Song B, Han J, Hassler J. The unfolded protein response is required to maintain the integrity of the endoplasmic reticulum, prevent oxidative stress and preserve differentiation in beta-cells. Diabetes Obes Metab. 2010;12 Suppl 2:99-107. https://doi. org/10.1111/j.1463-1326.2010.01281.x

PMid:21029306

32. Gilbert LA, Larson MH, Morsut L, Liu Z, Brar GA, Torres SE, et al. CRISPR-mediated modular RNA-guided regulation of transcription in eukaryotes. Cell. 2013;154(2):442-51. https:// doi.org/10.1016/j.cell.2013.06.044

PMid:23849981

33. Hendel A, Fine EJ, Bao G, Porteus MH. Quantifying on- and offtarget genome editing. Trends Biotechnol. 2015;33(2):132-40. https://doi.org/10.1016/j.tibtech.2014.12.001

PMid:25595557 